\title{
Juvenile Myelomonocytic Leukemia
}

National Cancer Institute

\section{Source}

National Cancer Institute. Juvenile Myelomonocytic Leukemia. NCI Thesaurus. Code C9233.

A myelodysplastic/myeloproliferative neoplasm of childhood that is characterized by proliferation principally of the granulocytic and monocytic lineages. Myelomonocytic proliferation is seen in the bone marrow and the blood. The leukemic cells may infiltrate any tissue, however liver, spleen, lymph nodes, skin, and respiratory tract are the most common sites of involvement. (WHO, 2001) 